\title{
Recent Approaches against SARS-COV-2: The Ongoing Outbreak of Coronavirus Disease 2019 (COVID-19)
}

\section{Manas Chakraborty ${ }^{1}$, Indranil Chatterjee ${ }^{2 *}$, Suman Kumar Nath ${ }^{2}$, Nilayan Guha ${ }^{3}$, Raksha Ray ${ }^{4}$ and Roushan Bhaskar ${ }^{4}$}

${ }^{1}$ Professor, Department of Pharmaceutical Biotechnology, Calcutta Institute of Pharmaceutical Technology and Allied Health Sciences, Howrah, West Bengal, India

${ }^{2}$ Assistant Professor, Birbhum Pharmacy School, Birbhum, West Bengal, India

${ }^{3}$ Assistant Professor, Bharat Technology, Howrah, West Bengal, India

${ }^{4}$ B. Pharm, P.G Institute of Medical Sciences, Chandrakona Town, Paschim Midnapore, West Bengal, India

*Corresponding Author: Indranil Chatterjee, Assistant Professor, Department of Microbiology, Birbhum Pharmacy School, Birbhum, West Bengal, India.

DOI: 10.31080/ASMI.2020.03.0649
Received: June 01, 2020

Published: July 11, 2020

(C) All rights are reserved by Indranil

Chatterjee., et al.

\begin{abstract}
Novel extreme intense respiratory condition coronavirus 2 (SARS-CoV-2) lies behind the continuous episode of coronavirus malady 2019 (COVID-19). There is a developing comprehension of SARS-CoV-2 in virology, the study of disease transmission, and clinical administration systems. Be that as it may, no enemy of SARS-CoV-2 medication or antibody has been authoritatively affirmed because of the nonattendance of satisfactory proof. Researchers are dashing to build up a treatment for COVID-19. Late investigations have uncovered numerous alluring remedial choices, regardless of whether some of them stay to be additionally affirmed in thorough preclinical models and clinical preliminaries. In this minireview, we mean to sum up the refreshed potential methodologies against SARS-CoV-2. We stress that further endeavors are justified to build up the most secure and best methodology.
\end{abstract}

Keywords: COVID-19; SARS-CoV-2; Antiviral Drugs; Treatment; Vaccines

Since December 2019, coronavirus infection 2019 (COVID-19) has been spreading around the globe, with more than 130,000 affirmed cases (starting at 13 March 2020) [1,2]. Extreme intense respiratory disorder coronavirus 2 (SARS-CoV-2), a novel betacoronavirus, is the causative specialist of this worldwide wellbeing danger [3]. Like different coronavirus strains, SARS-CoV-2 is portrayed by a round morphology with spike projections on the surface. It was shown that SARS-CoV-2 imparted high arrangement character to SARS-CoV and bat SARS-like coronavirus (SL-CoV) [4]. Remarkably, SARS-CoV-2 has lower pathogenicity than SARS-CoV yet higher transmissibility from human to human [5]. Cell section is the initial step of cross-species transmission. SARS-CoV-2 is bound to taint lung type II alveolar cells, which may clarify the serious alveolar harm after contamination [6].

The fast spread of COVID-19 has brought about a dire prerequisite for viable remedial procedures against SARS-CoV-2. At first, without authorized antibodies or affirmed antiviral medications, COVID-19 treatment was for the most part dependent on the experience of clinicians. The most up to date rule distributed by Na- 
tional Health Commission (NHC) of the Individuals' Republic of China suggests alpha interferon (IFN-), lopinavir/ritonavir, ribavirin, chloroquine phosphate, and arbidol as antiviral treatment [7]. Until this point in time, numerous potential methodologies have been uncovered dependent on the advancement of SARS-CoV-2 research, counting hindrance of SARS-CoV-2 combination/section, disturbance of SARS-CoV-2 replication, concealment of over the top provocative reaction, recovering plasma treatment, and the utilization of antibodies just as the mix of conventional Chinese medication and Western medication (As summed up in figure 1). Also, a number of clinical preliminaries are in progress to test the wellbeing and viability of competitor drugs. In this review, we sum up the present information on the potential treatment against SARSCoV-2 dependent on the developing fundamental and clinical information.

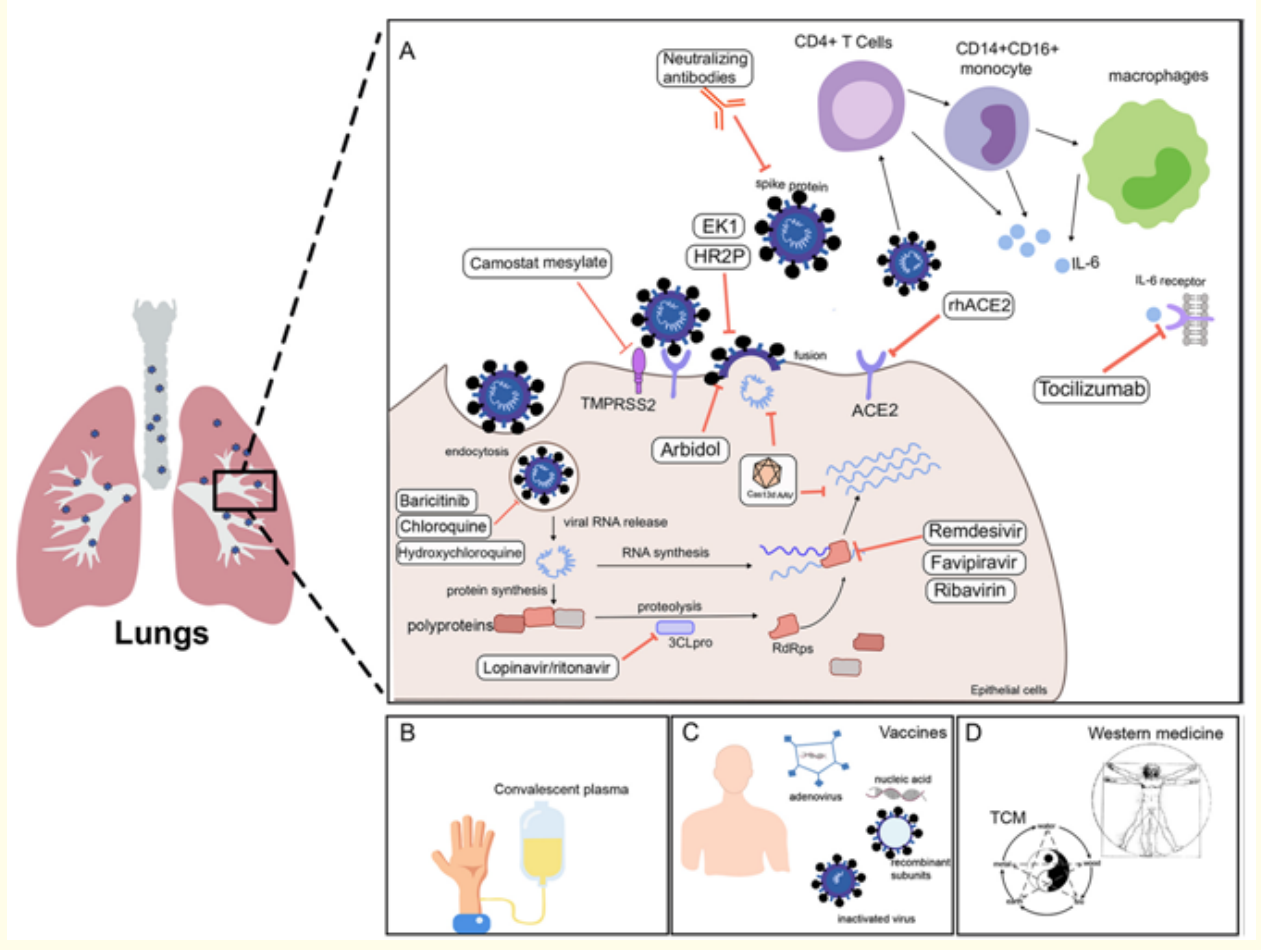

Figure 1: Updated potential approaches against SARS-CoV-2. (A) Hampering of SARS-CoV-2 entry; disruption of SARS-CoV-2 replication; inhibition of excessive inflammatory response. (B) Convalescent plasma treatment. (C) Vaccines. (D) Combinations of traditional Chinese and Western medicine. ACE2: Angiotensin-Converting Enzyme 2; rhACE2: Recombinant Human ACE2; HR2P: Heptad Repeat 2-Derived Peptides; EK1: A Modified OC43-HR2P Peptide; 3CLpro: 3C-Like Protease; RdRps: RNA-Dependent RNA Polymerases; AAV: Adeno-Associated Virus; IL-6: Interleukin-6; TCM: Traditional Chinese Medicine.

Updated attractive approaches against SARS-CoV-2: COVID-19 Entry of SARS-CoV-2 Inhibition

So also, to SARS-CoV, SARS-CoV-2 employments spike (S) protein to pick up section into have cells [8]. It was indicated that the $S$ protein on the surface of SARS-CoV-2 cell bound the section recep- tor angiotensin-changing over chemical 2 (ACE2) on contaminated cells [9]. SARS-CoV-2 was anticipated to perceive human ACE2 more productively than SARS-CoV [10]. In this way, focusing on the cooperations among ACE2 and S protein might be a potential methodology. 
In particular, a receptor restricting space (RBD) inside the $S$ protein is the basic objective for killing antibodies. Since SARS-CoV-2 S protein showed high homology to that of SARS-CoV, the accessible killing counter acting agent of SARS-CoV CR3022 was found to tie strongly with SARS-CoV-2 RBD [4].

In any case, Zheng and Song as of late announced that over $85 \%$ of the RBD counter acting agent epitopes in SARS-CoV-2 indicated amazing changes contrasted and SARSCoV, showing the need to grow new monoclonal antibodies for SARS-CoV-2 [11]. Moreover, the method of reasoning for the decision of the ACE2 receptor as a particular objective has been evaluated somewhere else [12,13].

Eminently, an open-mark, randomized, controlled, pilot clinical preliminary is in progress, further exploring the impact of recombinant human ACE2 (rhACE2; GSK2586881) in patients with extreme COVID-19 (Clinical Trials enrollment no. NCT04287686). It was proposed that $\mathrm{S}$ protein-determined cell section relied upon not just ACE2 yet in addition on the host cell serine protease TMPRSS2 [14].

Camostat mesylate, a clinically demonstrated inhibitor of TMPRSS2, fundamentally diminished lung cell line disease with SARSCoV-2 and could be considered for COVID-19 treatment [14]. In expansion, heptad rehash 1 (HR1) and heptad rehash 2 (HR2) on SARS-CoV-2 included viral and cell film combination [15]. Xia., et al. detailed that HR2-determined peptides (HR2P) and EK1 (a changed OC43-HR2P peptide) showed successful combination inhibitory action toward SARS-CoV-2 and would go about as combination/passage inhibitors to treat SARSCoV-2 contamination. Further examinations are justified to validate these ideas.

In addition, it was recommended that coronavirus section likewise included $\mathrm{pH}$-and receptor subordinate endocytosis $[16,17]$. Focusing on endocytosis might be another alternative for battling SARS-CoV-2. AP-2-related protein kinase 1 (AAK1) is a host kinase that controls clathrin-interceded endocytosis [18]. A gathering of endorsed drugs focusing on AAK1 were looked out dependent on man-made reasoning (AI) innovation [19]. Among them, the Janus kinase inhibitor baricitinib, an AAK1-restricting medication, was required to be a reasonable competitor medicate for COVID-19 in light of the fact that the standard treatment portions of baricitinib were adequate to restrain AAK1 [19].
Arbidol and chloroquine phosphate have been added to the rundown of potential treatment choices in the NHC rule for COVID-19 treatment [7]. Arbidol was appeared to restrain various encompassed infections by hindering infection passage/combination of viral layers with cell films [20]. Chloroquine, a conventional antimalarial tranquilize, was demonstrated to be powerful against SARS-CoV-2 contamination in vitro [21]. A few clinical preliminaries are in progress to test the adequacy and security of chloroquine phosphate against COVID-19 [22]. Results from in excess of 100 patients gave the main proof that chloroquine phosphate was increasingly compelling in hindering the worsening of pneumonia than control treatment [22]. Furthermore, Yao., et al. discovered that hydroxychloroquine (half compelling fixation [EC50] $0.72 \mathrm{M}$ ) was progressively strong as for repressing SARS-CoV-2 than chloroquine (EC50 5.47 M) in vitro [23].

In particular, the sub-atomic instrument of chloroquine phosphate in the treatment of COVID-19 remains tricky. It has been accounted for that chloroquine could hinder endosome-intervened viral passage or the late phases of viral replication [24]. More endeavors are expected to nail down the careful component.

\section{Restriction of SARS-Cov-2 replication}

Numerous antiviral specialists have been created against viral proteases, polymerases, MTases, and passage proteins. Clinical preliminaries are at present in progress to test various antiviral medications, for example, remdesivir (Clinical- Preliminaries enrollment no. NCT04252664 and NCT04257656), favipiravir (Chinese Clinical Trial enrollment no. ChiCTR2000029600 and ChiCTR2000029544), ASC09 (ChiCTR2000029603), lopinavir/ ritonavir (ChiCTR2000029387, ChiCTR2000029468, and ChiCTR2000029539), what's more, arbidol (ChiCTR2000029621). Martinez revealed that the most encouraging antiviral for battling SARS-CoV-2 was remdesivir [25].

Remdesivir is a monophosphoramidate prodrug of an adenosine simple. Its dynamic structure can consolidate into early popular RNA by the movement of RNA-subordinate RNA polymerases (RdRps), which at that point causes RNA combination capture [26]. Wang., et al. shown that remdesivir viably restrained SARS-CoV-2 in vitro [21]. The clinical state of the patient with the primary instance of COVID-19 affirmed in the United States improved after intravenous remdesivir organization [27]. Correspondingly, favipiravir and ribavirin are monophosphoramidate prodrugs of guanine 
analogs and have been endorsed for treatment of diseases by some different infections [28]. Notwithstanding, their antiviral impact in patients with COVID-19 needs thorough information to help their utilization. Lopinavir and ritonavir are protease inhibitors focusing on the coronavirus primary proteinase (3C-like protease; 3CLpro).

3CLpro is capable for handling the polypeptide interpretation item from the genomic RNA into the protein parts [29]. Highthroughput screening was likewise used to screen small molecule drugs focusing on the viral primary protease in clinical medication libraries [30]. Four atoms, including prulifloxacin, tegobuvir, bictegravir, and nelfinavir, demonstrated sensible restricting adaptations with the viral fundamental protease [30]. Focusing on the RNA genome of SARS-CoV-2 might be another methodology. Nguyen., et al. demonstrated the utilization of the novel CRISPR/ Cas13 RNA knockdown framework in severing the SARS-CoV-2 RNA genome [31]. This CRISPR/Cas13d framework was made out of a Cas13d protein and guide RNA-containing spacer succession explicitly correlative to the infection RNA genome. It was proposed that the Cas13d effector could be conveyed by means of an adenorelated infection (AAV) to the lung contaminated with SARS-CoV-2 [31].

\section{Excessive inflammatory response suppression}

An organized cytokine reaction is fundamental for the host resistant reaction. Notwithstanding, a dysregulated reaction prompts a hyperinflammatory condition in certain patients contaminated with SARS-CoV-2. It was accounted for that patients in escalated care units (ICUs) had higher grouping of cytokines in plasma than non-ICU patients with COVID-19, proposing that the cytokine storm was related with malady seriousness [32]. Furthermore, higher rates of granulocyte-macrophage province animating variable positive (GM-CSF_) and interleukin-6-positive (IL-6_) CD4_ $\mathrm{T}$ cells were disengaged from ICU patients contaminated with SARSCoV-2 than from non-ICU patients [33]. Taking into account this, restraint of over the top fiery reaction may speak to an aide treatment for COVID-19.

Nevertheless, the helpful utilization of corticosteroids, which has indicated astounding pharmacological impacts as for smothering rich and broken precise aggravation, is still disputable [25,32]. The current NHC rule underscores that the normal use of efficient corticosteroids isn't suggested except if shown for another explanation. In line, there were no accessible information demonstrating that patients profited by corticosteroid treatment in SARS-CoV or Middle East respiratory condition coronavirus (MERSCoV) contamination, which may be inferable from the concealment of resistant reaction against infection [34]. Eminently, an ongoing review study indicated the potential advantages accumulating from low-portion corticosteroid treatment in a subset of basically sick patients with SARS-CoV-2 [35]. More examinations are expected to discover how and when to utilize corticosteroids appropriately.

At the cell level, Zhou., et al. exhibited that CD4_ T cells were quickly actuated to deliver GM-CSF and other fiery cytokines after SARS-CoV-2 disease, which further incited CD14_CD16_monocyte actuation with significant levels of articulation of interleukin 6 (IL6) [33]. Hence, blocking GM-CSF or IL-6 receptor would conceivably diminish immunopathology brought about by SARS-CoV-2. In line, a multicenter, randomized, controlled clinical preliminary is in progress to inspect the adequacy and wellbeing of tocilizumab (an IL-6 receptor-explicit immune response) in patients with COVID-19 (Chinese Clinical Trial enrollment no. ChiCTR2000029765). Additionally, Fu., et al. referenced conceivable systems of SARS-CoV2-intervened incendiary reactions in which the killing antibodies activated $\mathrm{Fc}$ receptor (FCR)- interceded provocative reactions and intense lung injury [36]. Different choices to square FcR initiation may decrease SARS-CoV-2-actuated incendiary reactions [36].

\section{Convalescent plasma (CP) therapy}

With diseases for which there is no particular treatment accessible, treatment with gaining strength plasma has been proposed as a head treatment [37]. The CP is acquired from a benefactor who has recuperated from contamination by creating humoral resistance against the SARS-CoV-2 [38]. The defensive also, restorative advantage of $\mathrm{CP}$ was ascribed to the conceivable wellspring of explicit antibodies of human cause [39]. Be that as it may, assessment of the viability of CP treatment is still troublesome due to the absence of top notch randomized clinical preliminaries and of information of the exact instrument of activity of plasma treatment. As indicated by the NHC rule, the CP of recouped patients is for the most part utilized for patients in fast malady movement or in a serious or basic condition [40]. A few clinical preliminaries researching the viability and security of improving plasma transfusion in patients with COVID-19 are in progress (Chinese Clinical Trial enrollment no. ChiCTR2000030010, ChiCTR2000030179, and ChiCTR2000030381). 


\section{Vaccines}

With the worldwide spread of SARS-CoV-2, inoculation must be the most proficient and financially savvy intends to forestall and control COVID-19 [41]. Vigorous research endeavors are in progress to encourage the advancement of immunizations against SARSCoV-2. In particular, the $\mathrm{S}$ protein of SARS-CoV-2 stays a key objective for antibody advancement. As of late, Wrapp., et al. announced and shared the cryo-electron microscopy (cryo-EM) structure of SARS-CoV-2 S trimer, which empowered extra protein building endeavors and speeded up the procedure of immunization advancement [42]. What's more, Lucchese looked the pentapeptides exceptional to SARS-CoV-2 by contrasting the viral what's more, the human proteomes and found that 107 human-remote pentapeptides were inserted in S protein [43]. Further, these $S$ protein pentapeptides yielded 66 epitopes for antibody advancement [43]. In addition, since there were barely any accessible immunological examinations identified with SARS-CoV-2, Ahmed., et al. screened the SARS-CoV derived epitopes because of its elevated level of hereditary likeness with SARS-CoV-2 [44].

A screened set of SARS-CoV-determined B cell and T cell epitopes that mapped indistinguishably from SARS-CoV-2 proteins were distinguished, which would help the underlying period of immunization advancement [44]. In excess of 15 potential immunization contender for treatment of COVID-19 contamination are being created the world over, including inactivated immunization, recombinant subunits immunization, nucleic corrosive based antibody, adenoviral vector immunization, recombinant flu viral vector immunization, and so forth [45]. On 23 January 2020, the Coalition for Epidemic Preparedness Advancements (CEPI) reported the finding on DNA, mRNA, and "sub-atomic brace" antibody stages [46]. There was no current writing on SARS-CoV-2 immunization preliminaries starting at 13 March 2020. The security of immunization stays a top need for antibody improvement.

Combination therapy with traditional Chinese and western medicines

It was accounted that the Chinese medication items which were utilized to treat respiratory tract irresistible infections may be useful for SARS-CoV-2 treatment $[47,48]$. Among these items, Lianhua Qingwen cases and ShuFeng JieDu containers were appeared to apply autonomous antiviral impacts and synergistic antiviral impacts with Western medication items on flu infections, separately $[49,50]$. The most recent treatment rule in China included custom- ary Chinese medication (TCM) as one of the treatment choices for COVID-19. Wang., et al. revealed four cases with COVID-19 which demonstrated improvement after the patients were given joined Chinese and Western medication treatment [51]. Be that as it may, there are hardly any distributed examinations on Chinese medication items in the treatment of COVID-19 and, specifically, a lack of top notch looks into. Extra imminent, thorough populace contemplates are earnestly required to affirm the restorative impact of TCM. The component of their antiviral activity should be additionally lit up.

\section{Conclusion}

The potential restorative systems referenced above depend on the refreshed inquire about information for SARS-CoV-2. Among those choices, we foresee that the restorative drugs that legitimately target SARS-CoV-2 will be best. In addition, antibodies are basic for the avoidance and restriction of COVID-19 transmission. Remarkably, the empowering progresses in translating SARS-Cov-2 will prompt extra potential restorative targets. Further, solid preclinical and clinical investigations are expected to decide the sheltered and successful treatment for COVID-19.

\section{Acknowledgment}

All of us declare that we have no conflict of interest.

\section{Bibliography}

1. Guan WJ., et al. "Clinical characteristics of coronavirus disease 2019 in China". The New England Journal of Medicine (2020).

2. World Health Organization. Coronavirus disease 2019 (COVID-19) situation report-53 (2020).

3. Zhu N., et al. "A novel coronavirus from patients with pneumonia in China, 2019". The New England Journal of Medicine 382 (2020): 727-733.

4. Tian X., et al. "Potent binding of 2019 novel coronavirus spike protein by a SARS coronavirus-specific human monoclonal antibody". Emerging Microbes and Infections 9 (2020): 382-385.

5. Li Q., et al. "Early transmission dynamics in Wuhan, China, of novel coronavirus-infected pneumonia". The New England Journal of Medicine (2020).

6. Zhao YZZ., et al. "Single-cell RNA expression profiling of ACE2, the putative receptor of Wuhan 2019-nCov". BioRxiv (2020). 
7. National Health Commission of the People's Republic of China. Notice on printing and distributing the diagnosis and treatment plan of pneumonia with new coronavirus infection (trial version 7) (2020).

8. Walls AC., et al. "Structure, function, and antigenicity of the SARSCoV-2 spike glycoprotein". Cell (2020).

9. Zhou P., et al. "A pneumonia outbreak associated with a new coronavirus of probable bat origin". Nature 579 (2020): 270273.

10. Wan Y., et al. "Receptor recognition by novel coronavirus from Wuhan: an analysis based on decade-long structural studies of SARS”. Journal of Virology (2020).

11. Zheng M., et al. "Novel antibody epitopes dominate the antigenicity of spike glycoprotein in SARS-CoV-2 compared to SARSCoV". Cellular and Molecular Immunology (2020).

12. Zhang H., et al. "Angiotensin-converting enzyme 2 (ACE2) as a SARS-CoV-2 receptor: molecular mechanisms and potential therapeutic target". Intensive Care Medicine (2020).

13. Kruse RL. "Therapeutic strategies in an outbreak scenario to treat the novel coronavirus originating in Wuhan, China". F1000 Research 9 (2020): 72.

14. Hoffmann M., et al. "SARS-CoV-2 cell entry depends on ACE2 and TMPRSS 2 and is blocked by a clinically proven protease inhibitor". Cell (2020).

15. Xia S., et al. "Fusion mechanism of 2019-nCoV and fusion inhibitors targeting HR1 domain in spike protein". Cellular and Molecular Immunology (2020).

16. Inoue Y., et al. "Clathrin-dependent entry of severe acute respiratory syndrome coronavirus into target cells expressing ACE2 with the cytoplasmic tail deleted". Journal of Virology 81 (2007): 8722-8729.

17. Wang H., et al. "SARS coronavirus entry into host cells through a novel clathrin- and caveolae independent endocytic pathway". Cell Research 18 (2008): 290-301.

18. Neveu G., et al. "AP-2-associated protein kinase 1 and cyclin $\mathrm{G}$-associated kinase regulate hepatitis $\mathrm{C}$ virus entry and are potential drug targets". Journal of Virology 89 (2015): 43874404.

19. Richardson P., et al. "Baricitinib as potential treatment for 2019-nCoV acute respiratory disease". Lancet 395 (2020): e30- e31.
20. Blaising J., et al. "Arbidol as a broad-spectrum antiviral: an update". Antiviral Research 107 (2014): 84-94.

21. Wang M., et al. "Remdesivir and chloroquine effectively inhibit the recently emerged novel coronavirus (2019-nCoV) in vitro". Cell Research 30 (2020): 269-271.

22. Gao J., et al. "Breakthrough: chloroquine phosphate has shown apparent efficacy in treatment of COVID-19 associated pneumonia in clinical studies". Bioscience Trends (2020).

23. Yao X., et al. "In vitro antiviral activity and projection of optimized dosing design of hydroxychloroquine for the treatment of severe acute respiratory syndrome coronavirus 2 (SARSCoV-2)". Clinical Infection Disease (2020).

24. Savarino A., et al. "Effects of chloroquine on viral infections: an old drug against today's diseases?" Lancet Infection Disease 3 (2003): 722-727.

25. Martinez MA. "Compounds with therapeutic potential against novel respiratory 2019 coronavirus". Antimicrobe Agents Chemotherapy (2020).

26. Gordon CJ., et al. "The antiviral compound remdesivir potently inhibits RNA-dependent RNA polymerase from Middle East respiratory syndrome coronavirus". Journal of Biological Chemistry (2020).

27. Holshue ML., et al. "First case of 2019 novel coronavirus in the United States". The New England Journal of Medicine 382 (2020): 929-936.

28. Li G and De Clercq E. "Therapeutic options for the 2019 novel coronavirus (2019-nCoV)". Nature Review on Drug Discovery 19 (2020): 149-150.

29. Morse JS., et al. "Learning from the past: possible urgent prevention and treatment options for severe acute respiratory infections caused by 2019-nCoV". Chembiochemistry 21 (2020): 730-738.

30. Li YZJ., et al. "Therapeutic drugs targeting 2019-nCoV main protease by high throughput screening". BioRxiv (2020).

31. Nguyen TM., et al. "Virus against virus: a potential treatment for 2019-nCov (SARS-CoV-2) and other RNA viruses". Cell Research 30 (2020): 189-190.

32. Huang C., et al. "Clinical features of patients infected with 2019 novel coronavirus in Wuhan, China”. Lancet 395 (2020): 497506. 
33. Zhou YF, et al. "Aberrant pathogenic GM-CSF T cells and inflammatory CD14 CD16 monocytes in severe pulmonary syndrome patients of a new coronavirus". BioRxiv (2020).

34. Russell CD., et al. "Clinical evidence does not support corticosteroid treatment for 2019-nCoV lung injury". Lancet 395 (2020): 473-475.

35. Zhou W., et al. "Potential benefits of precise corticosteroids therapy for severe 2019-nCoV pneumonia". Signal Transduction and Targeted Therapy 5 (2020): 18.

36. Fu Y., et al. "Understanding SARS-CoV-2-mediated inflammatory responses: from mechanisms to potential therapeutic tools". Virologica Sinica (2020).

37. Chen L., et al. "Convalescent plasma as a potential therapy for COVID-19". Lancet Infection Disease (2020).

38. Garraud 0., et al. "Plasma therapy against infectious pathogens, as of yesterday, today and tomorrow". Transfusion Clinique et Biologique 23 (2016): 39-44.

39. Marano G., et al. "Convalescent plasma: new evidence for an old therapeutic tool?" Blood Transfusion 14 (2016): 152-157.

40. National Health Commission of the People's Republic of China. Notice on printing and distributing the convalescent plasma treatment for novel coronavirus pneumonia (trial version 2) (2020).

41. Lu S. "Timely development of vaccines against SARS-CoV-2". Emerging Microbes and Infections 9 (2020): 542-544.

42. Wrapp D., et al. "Cryo-EM structure of the 2019-nCoV spike in the prefusion conformation”. Science 367 (2020): 1260-1263.

43. Lucchese G. "Epitopes for a 2019-nCoV vaccine". Cellular and Molecular Immunology (2020).

44. Ahmed SF., et al. "Preliminary identification of potential vaccine targets for the COVID-19 coronavirus (SARS-CoV-2) based on SARS-CoV immunological studies". Viruses 12 (2020): E254.

45. Pang J., et al. "Potential rapid diagnostics, vaccine and therapeutics for 2019 novel coronavirus (2019-nCoV): a systematic review". Journal of Clinical Medicine (2020).

46. CEPI. CEPI to fund three programmes to develop vaccines against the novel coronavirus, ncov-2019 (2020).

47. Lu H. "Drug treatment options for the 2019-new coronavirus (2019-nCoV)". Bioscience Trends (2020).
48. Ren JL., et al. "Traditional Chinese medicine for COVID-19 treatment". Pharmacology Research 155 (2020): 104743.

49. Ding Y., et al. "The Chinese prescription lianhuaqingwen capsule exerts anti-influenza activity through the inhibition of viral propagation and impacts immune function". BMC Complementary and Alternative Medicine 17 (2017): 130.

50. Ji S., et al. "Unique synergistic antiviral effects of Shufeng Jiedu Capsule and oseltamivir in influenza A viral-induced acute exacerbation of chronic obstructive pulmonary disease". Biomed Pharmacotherapy 121 (2020): 109652.

51. Wang Z., et al. "Clinical characteristics and therapeutic procedure for four cases with 2019 novel coronavirus pneumonia receiving combined Chinese and Western medicine treatment". Bioscience Trends (2020).

\section{Assets from publication with us}

- Prompt Acknowledgement after receiving the article

- Thorough Double blinded peer review

- Rapid Publication

- Issue of Publication Certificate

- High visibility of your Published work

Website: www.actascientific.com/

Submit Article: www.actascientific.com/submission.php

Email us: editor@actascientific.com

Contact us: +919182824667 